\title{
Reusability of nanostencils for the patterning of Aluminum nanostructures by selective wet etching
}

\author{
O. Vázquez-Mena *, G. Villanueva, M.A.F. van den Boogaart, V. Savu, J. Brugger \\ Microsystems Laboratory, Ecole Polytechnique Fédérale de Lausanne (EPFL), Lausanne, 1015, Switzerland
}

Received 8 October 2007; received in revised form 16 December 2007; accepted 27 December 2007

Available online 20 January 2008

\begin{abstract}
One of the major advantages of stencil lithography is the possibility to use stencils many times. However, when stencils contain nanoapertures, the clogging of the membranes limits the useful life time of the stencils. The clogging is due to the accumulation of material deposited inside the apertures of the stencil. Here, we report a study on the effect of the clogging on the life time of stencils after $\mathrm{Al}$ depositions through the stencils. Then we present a method to clean the stencils based on Al wet etching to eliminate the clogging. We show that this method allows the reusability of stencils for the repeatable depositions of Al nanostructures.
\end{abstract}

(C) 2008 Published by Elsevier B.V.

Keywords: Stencil lithography; Clogging; Cleaning; Shadow mask; Nanostencils

\section{Introduction}

Stencil lithography (SL) is a shadow mask based technique that offers sub-micron deposition resolution for different materials and substrates $[1,2]$. Materials like metals [3], magnetic materials [4], organic molecules [5] and substrates like CMOS circuits [6], MEMS [1] and SAM's [7] have been patterned using stencils. An important advantage of SL is the possibility to reuse stencils many times. However, when stencils contain nanometric apertures, the useful life of stencils is limited due to the clogging of the membranes after material deposition $[2,8]$.

The clogging is a consequence of the accumulation of material in the stencils, reducing the size of the apertures as illustrated in Fig 1. For micrometric apertures, the clogging is not a major problem since the size reduction is very small compared to the size of the apertures. However, in the case of nanostencils, this problem becomes relevant since the thickness of material deposited $(10-100 \mathrm{~nm})$ is similar to the membrane thickness $(100-200 \mathrm{~nm})$ and the

\footnotetext{
* Corresponding author. Tel.: +41216936741; fax: +41216936670.

E-mail addresses: oscar.vazquez@epfl.ch (O. Vázquez-Mena), juergen.brugger@epfl.ch (J. Brugger).
}

aperture size (50-200 nm). This limits the life time of stencils and the repeatability of the deposited structures since the size of the apertures is reduced after each evaporation. Since the fabrication of nanostencils implies the use of high resolution techniques such as FIB [9], DUV [3] or E-Beam lithography [2], it is also highly convenient to extend the useful life time of the stencils and keep the size and quality of the apertures as long as possible.

As a solution, self-assembled monolayers have been reported [10] to reduce the effect of clogging, but eventually the stencils apertures get closed. The use of wet etching to eliminate the clogging of stencils after gold evaporation has been reported as well [11]. In this work, we present a cleaning method based on wet etching of $\mathrm{Al}$ that does not harm the membranes and we prove the long term usage of stencils for $\mathrm{Al}$ depositions of nanostructures.

\section{Nanostencil fabrication and material deposition}

The stencils used contain $100 \mathrm{~nm}$ thick low stress silicon nitride membranes. Their detailed fabrication can be found elsewhere $[9,12]$. The membranes are reinforced with corrugations $(2 \mu \mathrm{m}$ high $)$ that decrease the accumulated stress, 
Initial aperture

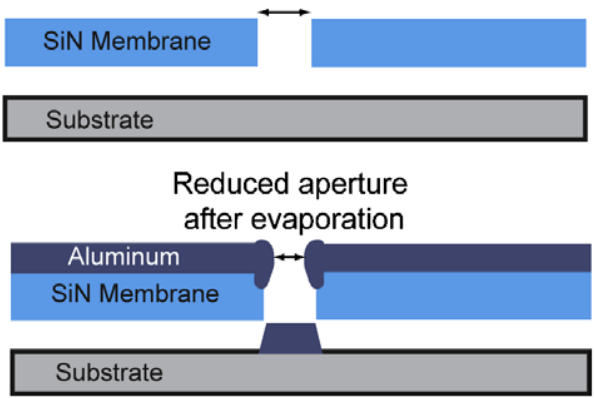

Fig. 1. Schematics of clogging. Top: stencil aperture before material deposition. Bottom: aperture size reduced due to the accumulation of material on the membrane (dimensions in the diagram not in scale).

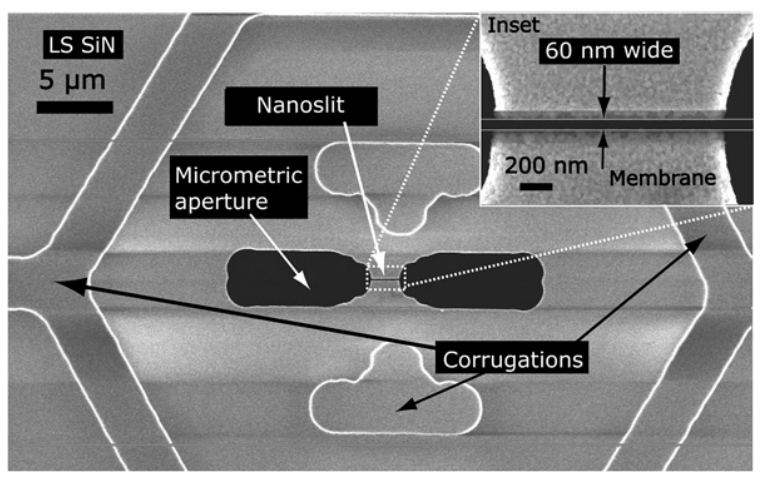

Fig. 2. Nanostencil aperture. SEM-image of a $100 \mathrm{~nm}$ thick LS-SiN stabilized membrane containing corrugations, micrometric apertures and a nanoslit. Inset: close up of the $60 \mathrm{~nm}$ wide slit.

making them more stable. Once the stencil is finished, we use FIB milling to fabricate the nanoslits on the membranes. Before FIB milling, we deposit a thin film of $25 \mathrm{~nm}$ of Al to avoid electrical charging during FIB milling. The SEM micrograph of a stencil aperture is shown in Fig. 2.

For metal deposition using stencil lithography, the stencil and substrate are clamped and then mounted into an evaporator for the $\mathrm{Al}$ deposition. For this experiment we have used a $100 \mathrm{~mm}$ (diameter) full wafer stencil and a $100 \mathrm{~mm}$ (diameter) silicon wafer with a $100 \mathrm{~nm}$ thick $\mathrm{SiO}_{2}$ layer as a substrate. The gap between the stencil membrane and the substrate ranges between 10 and $20 \mu \mathrm{m}$. This gap is due to the wafer curvatures of both stencil and substrate. The corrugations to stabilize the stencils do not contribute to this gap since they are recessed from the side of the stencil facing the substrate.

\section{Clogging}

In order to study the clogging of the membranes we performed three successive depositions of $\mathrm{Al}$ using the same stencil. We inspected the apertures with the SEM after their fabrication and then after each one of the three evaporations. For every evaporation we deposited $25 \mathrm{~nm}$ of Al.
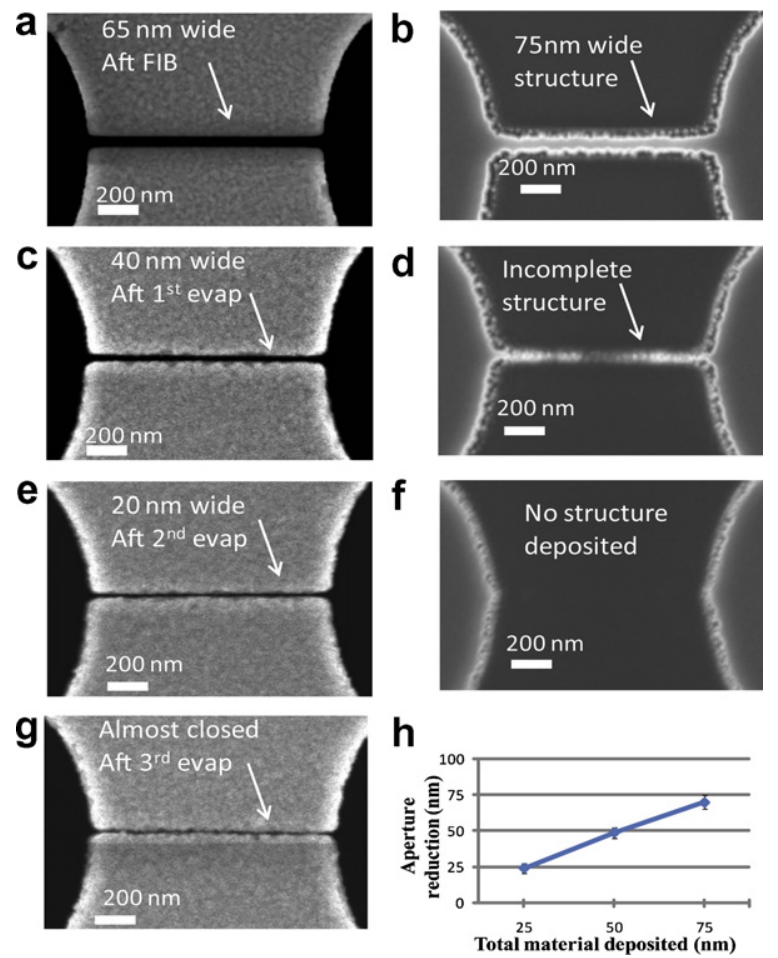

Fig. 3. Clogging and effect on deposited structures. (a-g) Stencil aperture and its corresponding deposited structures after successive evaporations showing the effect of clogging (details in Section 3). (h) Behavior of clogging as a function of material deposited.

Fig. 3 shows this series of experiments for the same aperture. Fig. 3a shows the stencil aperture after FIB milling and Fig. $3 b$ shows the deposited structure obtained from the first evaporation through the aperture shown in Fig. 3a. After the evaporation, the stencil was inspected (Fig. 3c) and a reduction in the size of the aperture was observed due to clogging. This stencil was then used to perform a second evaporation of $25 \mathrm{~nm}$ of Al. The obtained structure is shown in Fig. 3d and as observed it is incomplete. After this evaporation the stencil was again inspected (Fig. 3e) and the width of the aperture was further decreased. The stencil was used again for a third evaporation of $\mathrm{Al}$ ( $25 \mathrm{~nm}$ thick), however there was no structure deposited through the stencil aperture (Fig. 3f). This is probably due to the highly reduced amount of material passing through the clogged aperture that is not enough to define a structure on the substrate. The stencil aperture was inspected after this third evaporation (Fig. 3g) and it was almost closed due to the accumulation of Al. This shows that after the deposition of $75 \mathrm{~nm}$ of $\mathrm{Al}(25 \mathrm{~nm}$ for each of the three evaporations), this particular aperture was too clogged for further useful depositions.

In total, we studied 30 apertures with widths varying from $150 \mathrm{~nm}$ down to $60 \mathrm{~nm}$ after FIB milling. The results for the clogging are summarized in Fig. 3h. These measurements show that the size reduction of the nanoapertures due to clogging corresponds closely to the amount of material that is evaporated through the stencil. The smaller 
apertures $(\sim 60 \mathrm{~nm})$ get closed after the three evaporations described before, whereas the wider openings (initial width of $\sim 130 \mathrm{~nm}$ ) are reduced to $\sim 60 \mathrm{~nm}$ in width.

\section{Cleaning}

In order to remove the aluminum that accumulates on the stencil apertures, we have used a conventional aluminum wet etching solution (ANP) that is highly selective to silicon nitride. This prevents the damage or degradation of the membrane apertures. The composition of the etching solution is $\mathrm{CH}_{3} \mathrm{COOH}(100 \%), \mathrm{HNO}_{3}(70 \%)$ and $\mathrm{H}_{3} \mathrm{PO}_{4}$ $(85 \%)$ in proportions 5:3:75 and it is used at $35^{\circ} \mathrm{C}$. This "stencil cleaning" procedure is simple and consists in dipping the clogged stencil in the ANP solution during 20 min and rinsing it in water.

The stencil clogged with $75 \mathrm{~nm}$ of $\mathrm{Al}$ from the previous section was cleaned in the ANP solution as described before. The results are shown in Fig. 4. A stencil aperture after FIB milling is shown in Fig. 4a and the corresponding deposited structure is shown in Fig. 4b. After three evaporations (previous section) the stencil gets clogged and the aperture is almost closed as shown in Fig. 4c. The deposited structure through the clogged stencil is incomplete as well (Fig. 4d). Then the stencil was cleaned using the "stencil cleaning" procedure. Fig. 4e shows the stencil aperture after this process. As it is observed, the aperture does not suffer any damage or modification and more important, the stencil aperture recovers the original size after FIB milling. This cleaned stencil was used for the deposition of $25 \mathrm{~nm}$ of $\mathrm{Al}$ and the deposited structure (Fig. 4f) also recovers the size from the first deposition with the stencil (Fig. 4b).

The same stencil was used for diverse experiments and cleaned after each evaporation as described before. In this way, we assure that for every experiment each aperture maintains its size. So far, we have used the same stencil
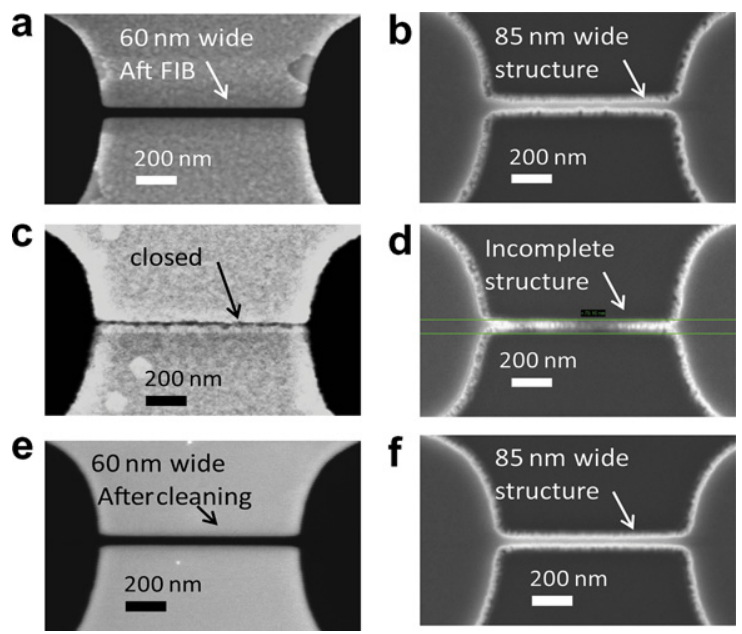

Fig. 4. Cleaning of stencils. Stencil and corresponding deposited structures for a new, clogged and cleaned stencil (details in Section 4).

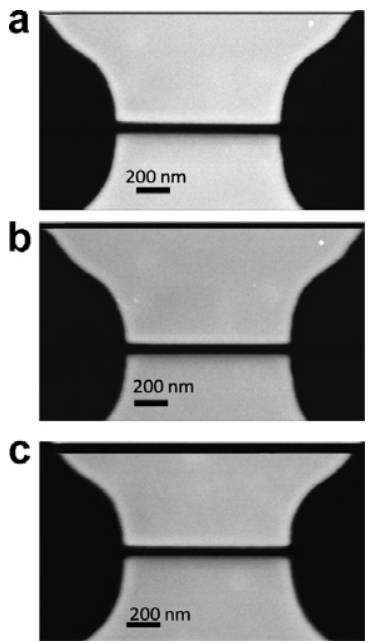

Fig. 5. Reusability of stencils. Images of the same stencil aperture after the 4th, 8th and 12th evaporation. In each case the stencil was cleaned after the evaporation.

12 times without observing any damage or degradation of the stencils meaning that we can continue using the same stencil. Fig. 5a-c shows images of the same aperture of the stencil after the 4th, 8th and 12th evaporation, respectively, being cleaned after each evaporation.

\section{Conclusions}

The clogging of the nanostencils reduces the useful life time of stencils by gradually closing the nanoapertures, limiting the reusability of stencils for the low-cost patterning of nanostructures. In the case of $\mathrm{Al}$, the aperture reduction corresponds closely to the amount of material deposited through the stencils. As an effective remedy, we have proven that by an ANP wet etching solution the stencils can be cleaned eliminating the aluminum that clogs the apertures. This solution has allowed the use of the same stencil 12 times for the patterning of sub-100 nm structures.

We have demonstrated that stencils can be reused and that SL can provide an effective low-cost technique for nanopatterning. Even though this study is only applied to aluminum, there are various different wet etch methods for metals that are highly selective to silicon nitride. This shows the potential of this technique as reliable and durable tool for the nanopatterning of diverse metals.

\section{Acknowledgments}

This effort is sponsored by the Swiss Federal Office for Science and Education (OFES) in the framework of the EC-funded FP6 project NaPa (NMP4-CT-2003-500120), the Swiss National Science Foundation, Project IC-Nano, 200021-112291/1 and the EPFL-STI Seed funding. The authors are very grateful to the EPFL Centre of MicroNano-Technology and to our colleagues at the Microsystems Laboratory. 


\section{References}

[1] J. Brugger, J.W. Berenschot, et al., Microelectronic Engineering 53 (2000) 403-405.

[2] M.M. Deshmukh, D.C. Ralph, M. Thomas, J. Silcox, Applied Physics Letters 75 (1999) 1631-1633.

[3] M.A.F. van den Boogaart, G.M. Kim, et al., Journal of Vacuum Science and Technology B 22 (2004) 3174-3177.

[4] A.F. Rodriguez, L.J. Heyderman, et al., Applied Physics Letters 89 (2006) 142508.

[5] S.M. Yi, S.H. Jin, J.D. Lee, C.N. Chu, Journal of Micromechanics and Microengineering 15 (2005) 263-269.
[6] J. Arcamone, M.A.F. van den Boogaart, F. Serra-Graells, S. Hansen, J. Brugger, F. Torres, G. Abadal, N. Barniol, F. Perez-Murano, International Electronic Devices Meeting 1-2 (2006) 250-253.

[7] E.A. Speets, P.T. Riele, et al., Advanced Functional Materials 16 (2006) 1337-1342.

[8] M. Lishchynska et al., Microelectronic Engineering 84 (2007) 42 53.

[9] G.M. Kim et al., Microelectronic Engineering 67-68 (2003) 609-614.

[10] M. Kolbel, R.W. Tjerkstra, et al., NanoLetters 2 (2002) 1339-1343.

[11] T.N. Tun, M.L. Win, et al., Nanotechnology 18 (2007) 335301.

[12] M.A.F. van den Boogaart, M. Lishchynska, et al., Sensors and Actuators A: Physical 130-131 (2006) 568-574. 